\title{
Bimetric cosmology is compatible with local tests of gravity
}

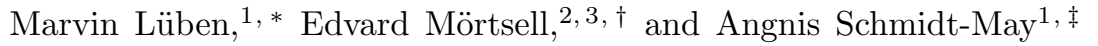 \\ ${ }^{1}$ Max-Planck-Institut für Physik (Werner-Heisenberg-Institut) \\ Föhringer Ring 6, 80805 Munich, Germany \\ ${ }^{2}$ Oskar Klein Centre, Stockholm University, AlbaNova University Center \\ 10691 Stockholm, Sweden \\ ${ }^{3}$ Department of Physics, Stockholm University, AlbaNova University Center \\ 10691 Stockholm, Sweden
}

\begin{abstract}
Recently, Kenna-Allison et al. claimed that bimetric gravity cannot give rise to a viable cosmological expansion history while at the same time being compatible with local gravity tests. In this note we review that claim and combine various results from the literature to provide several simple counter examples. We conclude that the results of Kenna-Allison et al. cannot hold in general.
\end{abstract}

\section{INTRODUCTION}

Ghost-free bimetric gravity is an extension of general relativity (GR) which describes the nonlinear interactions of a massive spin-2 field in a dynamical gravitational background [1, 2]. In contrast to its cousin massive gravity 3], it contains a massless spin-2 mode that mediates a long-range force and it possesses a smooth GR limit 1 .

In the linear theory of a massive spin-2 field coupled to a source, the zero-mass limit is discontinuous [8, 9]. For a single massive graviton, this discontinuity is in conflict with observations but can be cured by nonlinear interactions, a feature known as the Vainshtein mechanism [10]. In massive gravity, without the massless spin-2 mode, this mechanism is absolutely crucial for the phenomenological viability of the theory. In bimetric theory, the situation is fundamentally different since the gravitational interaction is mediated by a combination of the massive and the massless spin-2 mode. For large spin-2 masses, bimetric gravity passes all local gravity tests due to the strong Yukawa suppression of the massive graviton mode in the gravitational potential in Newtonian approximation. In addition, the coupling of the massive spin- 2 mode to matter can be made arbitrarily small, in which case the gravitational force is effectively mediated purely by the massless field. As a consequence, bimetric theory does not necessarily require a working Vainshtein mechanism in order to be in agreement with observational data.

Furthermore, bimetric theory can give rise to cosmological solutions with accelerated expansion even in the absence of vacuum energy [11 13. These selfaccelerating solutions require the mass scale which is associated to the breaking of independent diffeomorphisms

\footnotetext{
* mlueben@mpp.mpg.de

$\dagger$ edvard@fysik.su.se

$\ddagger$ angnissm@mpp.mpg.de

1 An important additional point to consider is the Cauchy problem, which is known to be well-posed in GR, but still the subject of ongoing work within bimetric theory 4 -7.
}

of the two metrics to be on the order of the Hubble scale. This scale is not necessarily identical to the Fierz-Pauli mass of the massive spin-2 mode since the latter depends on a different parameter combination. In particular, the spin-2 mass also involves the parameter which controls the coupling of the massive spin-2 mode to the matter source.

The authors of Ref. 14 claimed that in bimetric theory it is not possible to bring a viable cosmological expansion history in agreement with local gravity tests. Implicitly, their argument involved two steps: (1) The assumption that local gravity tests can only be passed if the Vainshtein mechanism is at work, and (2) a working Vainshtein mechanism can be shown to contradict a viable background cosmology due to incompatible requirements on the parameters of the theory.

In the following we invalidate both of these steps in the argument by giving explicit counter-examples.

\section{SHORT REVIEW OF BIMETRIC GRAVITY}

We start by presenting the action of ghost-free bimetric theory for two metric tensors $g$ and $f[1,15,2$

$$
\begin{aligned}
& S=\frac{m_{g}^{2}}{2} \int \mathrm{d}^{4} x\left(\sqrt{-g} R(g)+\alpha^{2} \sqrt{-f} R(f)\right) \\
& -m^{2} m_{g}^{2} \int \mathrm{d}^{4} x V(g, f)+\int \mathrm{d}^{4} x \sqrt{-g} \mathcal{L}_{\mathrm{m}}(g, \Phi),
\end{aligned}
$$

where $m_{g}$ is the bare Planck mass of the metric $g$ and $\alpha$ measures the ratio to the bare Planck mass of $f . R(\bullet)$ denotes the Ricci curvature scalar for each metric. The two metric tensors interact via the potential,

$$
V(g, f)=\sqrt{-g} \sum_{n=0}^{4} \beta_{n} e_{n}\left(\sqrt{g^{-1} f}\right),
$$

\footnotetext{
${ }^{2}$ For a review on bimetric gravity, see Ref. [16].
} 
where $e_{n}$ denotes the $n$-th elementary symmetric polynomial of the matrix argument. The constants $\beta_{n}$ are free parameters of the theory, and $\beta_{0}$ and $\beta_{4}$ parametrize the bare cosmological constants for $g$ and $f$, respectively ${ }^{3}$

Varying the action with respect to $g$ and $f$ yields two sets of modified Einstein equations,

$$
\begin{aligned}
G_{\mu \nu}^{g}+m^{2} V_{\mu \nu}^{g} & =\frac{1}{m_{g}^{2}} T_{\mu \nu}, \\
\alpha^{2} G_{\mu \nu}^{f}+m^{2} V_{\mu \nu}^{f} & =0
\end{aligned}
$$

where $G^{g}$ and $G^{f}$ are the Einstein tensors of $g$ and $f$, respectively. The terms $V^{g, f}$ arising from variation of the potential were derived in Ref. [1, 18. Finally, the stress-energy tensor of matter is defined as,

$$
T_{\mu \nu}=\frac{-2}{\sqrt{-g}} \frac{\delta \sqrt{-g} \mathcal{L}_{\mathrm{m}}}{\delta g^{\mu \nu}}
$$

\section{MASS EIGENSTATES AND GRAVITATIONAL FORCE}

Bimetric gravity has a well defined mass spectrum around proportional backgrounds, where the metrics are conformally related as $\bar{f}=c^{2} \bar{g}$ with the real constant $c$, determined by [2],

$$
\begin{aligned}
& \alpha^{2}\left(c \beta_{0}+3 c^{2} \beta_{1}+3 c^{3} \beta_{2}+c^{4} \beta_{3}\right) \\
& \quad=\beta_{1}+3 c \beta_{2}+3 c^{2} \beta_{3}+c^{3} \beta_{4} .
\end{aligned}
$$

We consider small fluctuations around that background,

$$
g_{\mu \nu}=\bar{g}_{\mu \nu}+\delta g_{\mu \nu}, \quad f_{\mu \nu}=\bar{f}_{\mu \nu}+\delta f_{\mu \nu} .
$$

Plugging this ansatz into the Einstein equations and keeping only terms up to linear order in the fluctuations, one finds that a massless spin- 2 field, $\delta G_{\mu \nu}$, and a massive spin-2 field, $\delta M_{\mu \nu}$ propagate on the proportional background [1, 2]. The massive mode has a Fierz-Pauli mass,

$$
m_{\mathrm{FP}}^{2}=m^{2} \frac{1+\alpha^{2} c^{2}}{\alpha^{2} c^{2}} c\left(\beta_{1}+2 \beta_{2} c+\beta_{3} c^{2}\right) .
$$

The original metric fluctuations are linear combinations of both mass eigenstates,

$$
\begin{aligned}
& \delta g_{\mu \nu} \propto \delta G_{\mu \nu}-\alpha^{2} \delta M_{\mu \nu} \\
& \delta f_{\mu \nu} \propto \delta M_{\mu \nu}+c^{2} \delta G_{\mu \nu}
\end{aligned}
$$

where we omitted the overall normalization.

\footnotetext{
${ }^{3}$ Ref. [14] refers to $\alpha_{0}$ as the bare cosmological constant, which is incorrect, since matter loops renormalize $\beta_{0}$. For an explicit confirmation of this, see equation (2.12) of Ref. [17]. The parameter $\alpha_{0}$ is a combination of all five $\beta_{n}$ and the exact relations between the two parametrizations can be found, for instance, in Ref. 2].
}

When $\alpha \ll 1$, the fluctuation of the physical metric $g_{\mu \nu}$ is almost aligned with the massless excitation. Since matter fields couple to the metric perturbations $\delta g_{\mu \nu}$, we expect to recover GR for $\alpha \ll 1$ [19]. Clearly, in this parameter region there is no conflict with current observational data.

For instance, let us consider a spherically symmetric background [20 24]. The contribution to the Newtonian potential coming from the massless mode is a Coulomblike term, $\sim r^{-1}$, proportional to the inverse distance between the source and the test particle, while the massive mode contributes a Yukawa-term $\sim e^{-m_{\mathrm{FP}} r} / r$. Hence, from eq. 8a it follows that the coupling $\frac{1}{m_{g}^{2}} \delta g_{\mu \nu} T^{\mu \nu}$ will produce the following linearized gravitational potential,

$$
V(r)=-\frac{1}{m_{\mathrm{Pl}}^{2}}\left(\frac{1}{r}+\frac{4 \alpha^{2} c^{2}}{3} \frac{e^{-m_{\mathrm{FP} r}}}{r}\right),
$$

where the physical Planck mass is $m_{\mathrm{Pl}}^{2}=m_{g}^{2}\left(1+\alpha^{2} c^{2}\right)[2]$. Whenever the first term in the gravitational potential dominates over the second one, the solution behaves approximately like GR and does not require a Vainshtein mechanism. We can thus identify two parameter régimes in which GR is restored,

$$
\begin{aligned}
& \text { 1.) } \alpha c \ll 1, \\
& \text { 2.) } m_{\mathrm{FP}} \gg \ell^{-1} \text {, }
\end{aligned}
$$

where $\ell$ is the typical length scale of the system (e.g. $\ell \simeq 1 \mathrm{AU}$ for the Solar System) .

\section{EXAMPLE: SOLAR SYSTEM}

In this section, we demonstrate the general results discussed in the previous section in an explicit example: the Solar System. For concreteness, we derive numerical values for the Sun as central source of the gravitational potential. Not all Solar System tests are based on this scenario, so our arguments here should be viewed as qualitative. A detailed quantitative analysis is left for future work. Our findings are summarised in fig. 1 and fig. 2 .

\section{A. Yukawa suppression}

At the scale of $1 \mathrm{AU} \simeq 1.5 \times 10^{13} \mathrm{~cm}$, deviations from the inverse square law for the gravitational force are constrained to be $\lesssim 10^{-9}[25$. We aim at providing the most conservative constraints on the bimetric parameters. Hence, we use this bound (which is the most stringent one) on deviations for any distance from the Sun. Comparing the two contributions in $\sqrt{9}$, this requires 4

$$
\frac{4 \alpha^{2}}{3} e^{-m_{\mathrm{FP}} r} \lesssim 10^{-9}
$$

\footnotetext{
4 In this section, we absorb $c$ into $\alpha$ for simplicity. Reinserting $c$ does not change any of our findings.
} 


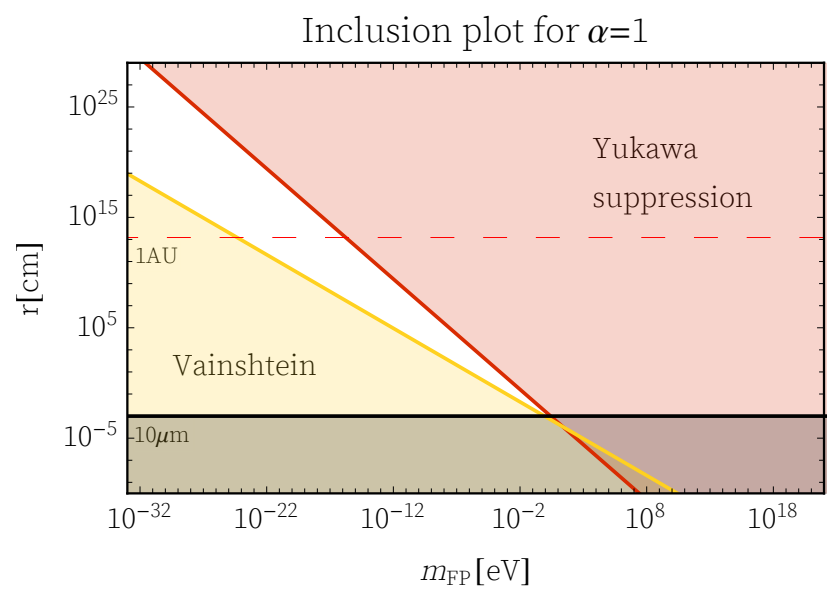

FIG. 1: For the Sun as central source, this figure indicates deviations from GR in the Newtonian force, for distances $r$ to the test particle as a function of the spin- 2 mass $m_{\mathrm{FP}}$. For large $m_{\mathrm{FP}}$ and large $r$, the contribution from the massive mode is sufficiently suppressed, yielding the red-shaded region. For small $m_{\mathrm{FP}}$ and small $r$, the Vainshtein mechanism restores GR, yielding the yellow-shaded region. Below $10 \mu \mathrm{m}$ no observational constraints exist.

Deviations due to the massive mode are arbitrarily small in both the previously identified parameter limits. The red line in fig. 1 represents the bound eq. 10 for the case $\alpha=1$. Therefore, in the red-shaded region, the Yukawalike term in the gravitational potential is always smaller than all observational bounds. A value for $\alpha$ smaller than unity makes the deviations even smaller.

Local gravity tests inside the Solar System provide strong constraints on deviations from GR down to scales of $\sim 10 \mu \mathrm{m}[25]$. As a very rough estimate, we use eq. (10) to define a critical spin-2 mass (for $\alpha \simeq 1$ ),

$$
m_{\text {crit }} \simeq 2.6 \mathrm{eV}
$$

above which no deviations from GR are detectable via observations in the Solar System. This is indicated by the black-shaded region in fig. 1 .

For $m_{\mathrm{FP}} \gg m_{\text {crit }}$ and in the red-shaded region of effective Yukawa suppression, the Vainshtein mechanism is certainly not needed to restore GR.

\section{B. Vainshtein regime}

Close to the source, nonlinear terms become as important as the linear ones in a way, such that GR is restored. This is the well-known Vainshtein mechanism [10] and it is active within a sphere defined by the Vainshtein radius,

$$
r_{V}=\left(\frac{r_{S}}{m_{\mathrm{FP}}^{2}}\right)^{1 / 3}
$$

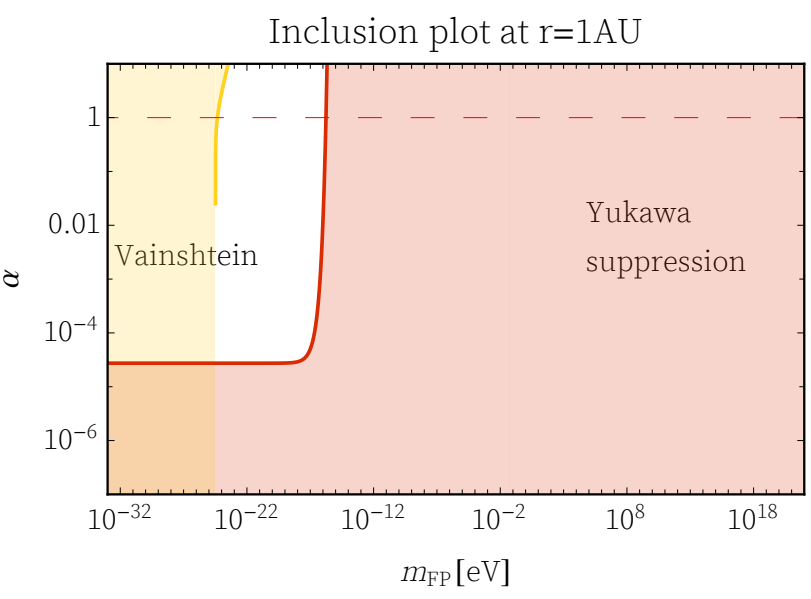

FIG. 2: This figure shows the allowed parameter regions in the $\alpha-m_{\mathrm{FP}}$-plane at the length scale $r=1 \mathrm{AU}$. For large $m_{\mathrm{FP}}$, the parameter $\alpha$ is not constrained to be small thanks to the Yukawa suppression in the potential. For small $m_{\mathrm{FP}}$, it is unconstrained due to the Vainshtein mechanism. Only for masses from $10^{-24}$ to $10^{-16} \mathrm{eV}$ observations require $\alpha \lesssim 10^{-5}$.

around an object of mass $M$ with Schwarzschild radius $r_{S}=\left(1+\alpha^{2}\right) M / m_{\mathrm{Pl}}^{2}[20,24$. Even though the expression derived in Ref. 14] seems to differ by a numerical factor (which is not manifestly positive) from (12), the scale is the same as long as the parameters satisfy $\beta_{n} \sim \mathcal{O}(1) 5^{5}$

Well inside the Vainshtein radius, deviations from the inverse-square law of the gravitational force scale like $\left(r / r_{V}\right)^{3}$ [20, 22] and hence, in order to satisfy (10), we require (for $\alpha \simeq 1$ ),

$$
\frac{r}{r_{V}} \lesssim 10^{-3}
$$

Choosing a solar mass object $M=M_{\odot}$ defines the orange line in fig. 1 as a rough estimate for the threshold of an effective Vainshtein mechanism. Consequently, well inside the yellow-shaded region, the gravitational force is certainly indistinguishable from GR for any bimetric parameters.

\section{Constraints on the spin-2 coupling}

Only in the region left white in fig. 1. significant deviations from GR could occur. However, we still have the free parameter $\alpha$, which we can use to suppress the extra term in the Newtonian potential. The most stringent

\footnotetext{
${ }^{5}$ We emphasize that the value for the Vainshtein radius is derived assuming $r<m_{\mathrm{FP}}^{-1}$. For the Solar System, this is not an issue since the intersection of the lines corresponding to $r_{V}$ and $m_{\mathrm{FP}}^{-1}$ lies close to the observable threshold of $\sim 10 \mu \mathrm{m}$. The region where $m_{\mathrm{FP}}^{-1} \sim r_{V}$ should be treated more carefully, but can definitely be brought in agreement with data by making $\alpha$ small.
} 
bound comes from close to the Vainshtein radius, where the observational bound 10 requires,

$$
\alpha \lesssim 10^{-5}
$$

see also fig. 2 Then, for any mass of the spin-2, deviations from the GR prediction are undetectable with current experimental precision.

For very small spin-2 masses, the Vainshtein radius of the Sun becomes larger than the Solar System itself. As a conservative estimate, evaluating eq. (13) at $r=100 \mathrm{AU}$ implies $m_{\mathrm{FP}} \lesssim 5 \times 10^{-28} \mathrm{eV}$. For smaller spin-2 masses the Kuiper belt is well inside the Vainshtein sphere and the Solar System does not constrain $\alpha$ to be small.

We conclude this section by stressing the following points: For a large spin- 2 mass, $m_{\mathrm{FP}} \gg m_{\text {crit }}$, the Vainshtein mechanism is not necessary for the theory to pass all Solar System tests since the Yukawa suppression of the contribution from the massive mode is too large. For very small masses, the Vainshtein mechanism has to be (and is) active. These results hold for any value of $\alpha \lesssim 1$. Only for intermediate spin- 2 masses the parameter $\alpha$ has to satisfy the bound (14).

\section{GRAVITATIONAL WAVES AND GALACTIC TESTS}

Constraints from gravitational waves were derived in Ref. [26], excluding a small region of the parameter space: Within the spin-2 mass range of $10^{-22}$ to $10^{-21} \mathrm{eV}$, the ratio of the Planck masses has to satisfy $\alpha \lesssim 0.3$. For a spin-2 mass outside that range, gravitational wave observations do not constrain $\alpha$ to be small.

On galactic scales, observations of galactic velocity dispersions and gravitational lensing can be used to constrain the bimetric parameters [23. For $m_{\mathrm{FP}} \simeq H_{0}$ and for a galaxy of mass $M=10^{12} M_{\odot}$, deviations from GR predictions are expected to be on the order of $\left(r / r_{\mathrm{V}}\right)^{3} \simeq 2 \times 10^{-13}[22$, which is within the observational bounds by a factor of $4 \times 10^{-12}$ [27, 28]. Data from cluster lensing requires $\alpha \lesssim 0.7$ for a spin- 2 mass range of $10^{-30}$ to $10^{-28} \mathrm{eV}$ [29], where neither the Vainshtein mechanism nor the Yukawa suppression restore GR on the length scales relevant to observations. Outside of this range, $\alpha$ is not constrained to be small since it is not needed to restore GR.

For a combined exclusion plot with galactic and extragalactic constraints as well as bounds from gravitational wave detection on the bimetric parameters, see fig. 9 in Ref. 29] ${ }^{6}$ We conclude that tests of the gravitational law outside the Solar System do not provide constraints on the bimetric parameter space in the region where $\alpha \lesssim 0.1$. Thus the most stringent bounds on bimetric gravity are

\footnotetext{
${ }^{6}$ Note that $\alpha=\tan \theta$ in the notation of Ref. 29] and their plot is linear in $\theta$.
}

those from the Solar System, discussed in the previous section.

\section{BIMETRIC COSMOLOGIES}

The equations of motion of bimetric gravity evaluated on a homogeneous and isotropic ansatz for both metrics give two sets of equations that can be combined into one modified Friedmann equation of the general form,

$$
H^{2}=F(\rho) \text {. }
$$

Here $H=\dot{a} / a$ is the Hubble function of the physical scale factor $a(t)$ inside the physical metric $g_{\mu \nu}$ and $\rho$ is the matter energy density, i.e., the 00-component of the perfect fluid source $T_{\nu}^{\mu}$.

In the following, we will assume that all $\beta_{n}$ parameters, are of order unity and the mass scale $m$ (not necessarily $m_{\mathrm{FP}}$ ) is of order $H_{0}$. For concreteness we will consider a simple bimetric model with parameters $\beta_{0}=\beta_{3}=\beta_{4}=0$. All of our findings straightforwardly generalize to models with generic $\mathcal{O}(1)$ values for all $\beta_{n}$.

\section{A. Large mass region}

In Ref. 19 it was shown that for $\alpha \ll 1$, the Friedmann equation 15 can be approximated by the simple form,

$$
H^{2}=\frac{\rho}{3 m_{\mathrm{Pl}}^{2}}-\frac{2 \beta_{1}^{2}}{3 \beta_{2}} m^{2}+\mathcal{O}\left(\alpha^{2}\right) .
$$

The Fierz-Pauli mass in eq. (7), defined at the de Sitter point is,

$$
m_{\mathrm{FP}}^{2}=m^{2}\left(1+\alpha^{-2} c^{-2}\right)\left(c \beta_{1}+2 c^{2} \beta_{2}\right) \sim \frac{m^{2}}{\alpha^{2}},
$$

where we have used that $c=-\frac{\beta_{1}}{3 \beta_{2}}+\mathcal{O}\left(\alpha^{2}\right) \sim \mathcal{O}(1)$ which follows from the background equation (5). Clearly $\alpha \ll 1$ implies that,

$$
m_{\mathrm{FP}} \gg H_{0} \text {. }
$$

This shows that we can easily be in the parameter regime where no Vainshtein mechanism is needed and at the same time have a valid background cosmology that matches exactly that of GR with cosmological constant $\Lambda=2 m^{2} \beta_{1}^{2} /\left|\beta_{2}\right|$. In fact, the approximation in eq. 16 is valid up to the scale $H \sim m_{\mathrm{FP}}$ [19]. By making the spin-2 mass large, we can thus push back the deviations from GR to arbitrary early times. Explicitly, already for a spin- 2 mass of $m_{\mathrm{FP}} \simeq 0.4 \mathrm{eV}$ the background expansion in this simple model follows (almost) exactly the $\Lambda$ CDM prediction until the CMB. At earlier times, when $H>m_{\mathrm{FP}}$, we expect $\mathrm{GR}$ to be recovered by nonlinear effects, as we shall discuss further below. Moreover, the cosmological evolution at early times is dominated by the matter density $\rho$ [13] and will therefore anyway follow almost exactly the evolution predicted by the $\Lambda \mathrm{CDM}$ model. 


\section{B. Small mass region}

Now, we consider the parameter region, where $\alpha \simeq 1$. With our assumption $\beta_{n} \sim \mathcal{O}(1)$, this implies

$$
m_{\mathrm{FP}} \simeq H_{0} .
$$

This parameter region has received a lot of attention in the literature, see e.g. Ref. [11-13, 30, 31]. In Ref. [30] various models with $\alpha=1$ were compared to observations, many of which give a good fit to data while still allowing for testable deviations from the standard $\Lambda \mathrm{CDM}$ scenario. The crucial point is that this parameter region is not in conflict with a successful Vainshtein mechanism around spherically symmetric sources. For a spin- 2 mass on the order of the Hubble rate today, the Vainshtein radius of the sun is $r_{V} \simeq 10^{22} \mathrm{~cm}$, which is on the order of the size of the Milky Way. Hence, no constraints on $\alpha$ exist for such a tiny spin- 2 mass. Lensing constraints do not provide bounds on the value of $\alpha$ for small spin-2 masses either [29], see section $\mathrm{V}$. The only caveat here is that the effective Planck mass in cosmological solutions must be matched with Newton's constant in the gravitational force law, which may (mildly) constrain one of the $\beta_{n}$ parameters.

\section{Cosmological perturbations}

Although bimetric gravity gives rise to a viable expansion history while passing all local gravity tests in both these parameter regions, the cosmological perturbations behave differently from GR. In particular, the FLRW background is not always stable against scalar fluctuations during the entire expansion history due to a gradient instability [32 43]. Ref. [19] showed that for times earlier than,

$$
H \simeq m_{\mathrm{FP}},
$$

the instability sets in. Thus, by making the spin-2 mass large (which is equivalent to $\alpha \ll 1$ in our parametrization), the instabilities can be pushed to arbitrarily early times.

Naïvely, these results seem to disfavour the parameter region with small spin-2 mass [40]. However, precisely when the Hubble rate exaggerates the spin-2 mass, the inset of the gradient instability simply implies that nonlin- ear effects become as important as linear ones, invalidating linear perturbation theory. The results of Ref. [44, 45] suggest that these nonlinear effects are in fact not problematic but instead restore GR at early times through the Vainshtein mechanism.

\section{CONCLUSION}

We have discussed on general grounds why bimetric theory for a large range of parameter values can give rise to a viable cosmology while at the same time passing all other tests of the gravitational force law.

In particular, we demonstrated explicitly that for a weak coupling of the massive spin- 2 mode to matter and a large spin-2 mass, bimetric theory becomes essentially indistinguishable from GR in cosmological solutions and on all distance scales in the galaxy.

We emphasize once more that this is in sharp contrast to massive gravity, which can be obtained as a parameter limit in bimetric gravity by sending $\alpha \rightarrow \infty$ and which requires the Vainshtein mechanism. For a summary of the much stronger constraints on massive gravity, see Ref. 46].

We also showed that even in the different regime of small spin-2 mass and Planck scale coupling for the massive spin-2, bimetric theory is compatible with all available observational data.

The assumptions in our explicit examples may seem restrictive. The theory has enough free parameters to achieve a large spin-2 mass even when $\alpha \simeq 1$ and conversely, a small spin- 2 mass $m_{\mathrm{FP}} \simeq H_{0}$ although $\alpha \ll 1$. These more general models, which require values for the $\beta_{n}$ parameters different from $\mathcal{O}(1)$, have several branches of solutions and the expressions can be lengthy. It would be interesting to study the resulting phenomenology, which we leave for future work. It is possible that the results of Ref. [14] apply to these parameter regions. Nevertheless, the examples we presented here clearly show that they do not hold in general.

We conclude that bimetric cosmologies are certainly not incompatible with local gravity tests.

\section{Acknowledgments}

This work is supported by a grant from the MaxPlanck-Society.
[1] S.F. Hassan and Rachel A. Rosen, "Bimetric Gravity from Ghost-free Massive Gravity," JHEP 1202, 126 (2012), arXiv:1109.3515 [hep-th]

[2] S.F. Hassan, Angnis Schmidt-May, and Mikael von Strauss, "On Consistent Theories of Massive Spin-2 Fields Coupled to Gravity," JHEP 1305, 086 (2013),
arXiv:1208.1515 [hep-th]

[3] Claudia de Rham, Gregory Gabadadze, and Andrew J. Tolley, "Resummation of Massive Gravity," Phys.Rev.Lett. 106, 231101 (2011), arXiv:1011.1232 [hep-th] 
[4] Mikica Kocic, "Geometric mean of bimetric spacetimes," (2018), arXiv:1803.09752 [hep-th].

[5] Mikica Kocic, "Causal propagation of constraints in bimetric relativity in standard $3+1$ form," (2018), arXiv:1804.03659 [hep-th].

[6] Mikica Kocic, Anders Lundkvist, and Francesco Torsello, "On the ratio of lapses in bimetric relativity," (2019), arXiv:1903.09646 [gr-qc]

[7] Francesco Torsello, Mikica Kocic, Marcus Högås, and Edvard Mörtsell, "Covariant BSSN formulation in bimetric relativity," (2019), arXiv:1904.07869 [gr-qc]

[8] H. van Dam and M.J.G. Veltman, "Massive and massless Yang-Mills and gravitational fields," Nucl.Phys. B22, 397-411 (1970)

[9] V.I. Zakharov, "Linearized gravitation theory and the graviton mass," JETP Lett. 12, 312 (1970).

[10] A.I. Vainshtein, "To the problem of nonvanishing gravitation mass," Phys.Lett. B39, 393-394 (1972).

[11] D. Comelli, M. Crisostomi, F. Nesti, and L. Pilo, "FRW Cosmology in Ghost Free Massive Gravity," JHEP 1203, 067 (2012), arXiv:1111.1983 [hep-th]

[12] Mikhail S. Volkov, "Cosmological solutions with massive gravitons in the bigravity theory," JHEP 1201, 035 (2012), arXiv:1110.6153 [hep-th]

[13] Mikael von Strauss, Angnis Schmidt-May, Jonas Enander, Edvard Mörtsell, and S.F. Hassan, "Cosmological Solutions in Bimetric Gravity and their Observational Tests," JCAP 1203, 042 (2012), arXiv:1111.1655 [gr-qc]

[14] Michael Kenna-Allison, A. Emir Gumrukcuoglu, and Kazuya Koyama, "On the viability of bigravity cosmology," (2018), arXiv:1812.05496 [hep-th].

[15] S.F. Hassan and Rachel A. Rosen, "Confirmation of the Secondary Constraint and Absence of Ghost in Massive Gravity and Bimetric Gravity," JHEP 1204, 123 (2012), arXiv:1111.2070 [hep-th].

[16] Angnis Schmidt-May and Mikael von Strauss, "Recent developments in bimetric theory," J. Phys. A49, 183001 (2016), arXiv:1512.00021 [hep-th].

[17] Claudia de Rham, Lavinia Heisenberg, and Raquel $\mathrm{H}$. Ribeiro, "On couplings to matter in massive (bigravity," Class. Quant. Grav. 32, 035022 (2015), arXiv:1408.1678 [hep-th].

[18] S.F. Hassan and Rachel A. Rosen, "On Non-Linear Actions for Massive Gravity," JHEP 1107, 009 (2011) arXiv:1103.6055 [hep-th].

[19] Yashar Akrami, S. F. Hassan, Frank Könnig, Angnis Schmidt-May, and Adam R. Solomon, "Bimetric gravity is cosmologically viable," Phys. Lett. B748, 37-44 (2015), arXiv:1503.07521 [gr-qc]

[20] Eugeny Babichev and Marco Crisostomi, "Restoring general relativity in massive bigravity theory," Phys. Rev. D88, 084002 (2013), arXiv:1307.3640 [gr-qc].

[21] D. Comelli, M. Crisostomi, F. Nesti, and L. Pilo, "Spherically Symmetric Solutions in Ghost-Free Massive Gravity," Phys.Rev. D85, 024044 (2012), arXiv:1110.4967 [hep-th]

[22] Jonas Enander and Edvard Mortsell, "On stars, galaxies and black holes in massive bigravity," JCAP 1511, 023 (2015), arXiv:1507.00912 [astro-ph.CO]

[23] Jonas Enander and Edvard Mörtsell, "Strong lensing constraints on bimetric massive gravity," JHEP 1310, 031 (2013), arXiv:1306.1086 [astro-ph.CO]]

[24] Eugeny Babichev, Luca Marzola, Martti Raidal, Angnis Schmidt-May, Federico Urban, Hardi Veermäe, and
Mikael von Strauss, "Heavy spin-2 Dark Matter," JCAP 1609, 016 (2016), arXiv:1607.03497 [hep-th].

[25] Clifford M. Will, "The Confrontation between General Relativity and Experiment," Living Rev. Rel. 17, 4 (2014), arXiv:1403.7377 [gr-qc]

[26] Kevin Max, Moritz Platscher, and Juri Smirnov, "Gravitational Wave Oscillations in Bigravity," Phys. Rev. Lett. 119, 111101 (2017), arXiv:1703.07785 [gr-qc].

[27] Josiah Schwab, Adam S. Bolton, and Saul A. Rappaport, "Galaxy-Scale Strong Lensing Tests of Gravity and Geometric Cosmology: Constraints and Systematic Limitations," Astrophys. J. 708, 750-757 (2010), arXiv:0907.4992 [astro-ph.CO]

[28] Stefan Sjors and Edvard Mortsell, "Spherically Symmetric Solutions in Massive Gravity and Constraints from Galaxies," JHEP 02, 080 (2013), arXiv:1111.5961 [gr-qc].

[29] Moritz Platscher, Juri Smirnov, Sven Meyer, and Matthias Bartelmann, "Long Range Effects in Gravity Theories with Vainshtein Screening," JCAP 1812, 009 (2018), arXiv:1809.05318 [astro-ph.CO]

[30] Yashar Akrami, Tomi S. Koivisto, and Marit Sandstad, "Accelerated expansion from ghost-free bigravity: a statistical analysis with improved generality," JHEP 1303, 099 (2013), arXiv:1209.0457 [astro-ph.CO].

[31] Frank Könnig, Aashay Patil, and Luca Amendola, "Viable cosmological solutions in massive bimetric gravity," JCAP 1403, 029 (2014), arXiv:1312.3208 [astro-ph.CO].

[32] D. Comelli, M. Crisostomi, and L. Pilo, "Perturbations in Massive Gravity Cosmology," JHEP 1206, 085 (2012), arXiv:1202.1986 [hep-th]

[33] Nima Khosravi, Hamid Reza Sepangi, and Shahab Shahidi, "Massive cosmological scalar perturbations," Phys.Rev. D86, 043517 (2012), arXiv:1202.2767 [gr-qc].

[34] Marcus Berg, Igor Buchberger, Jonas Enander, Edvard Mörtsell, and Stefan Sjörs, "Growth Histories in Bimetric Massive Gravity," JCAP 1212, 021 (2012), arXiv:1206.3496 [gr-qc].

[35] Matteo Fasiello and Andrew J. Tolley, "Cosmological Stability Bound in Massive Gravity and Bigravity," JCAP 1312, 002 (2013), arXiv:1308.1647 [hep-th].

[36] D. Comelli, M. Crisostomi, and L. Pilo, "FRW Cosmological Perturbations in Massive Bigravity," Phys.Rev. D90, 084003 (2014), arXiv:1403.5679 [hep-th].

[37] Antonio De Felice, A. Emir Gümrükçüoğlu, Shinji Mukohyama, Norihiro Tanahashi, and Takahiro Tanaka, "Viable cosmology in bimetric theory," JCAP 1406, 037 (2014), arXiv:1404.0008 [hep-th],

[38] Adam R. Solomon, Yashar Akrami, and Tomi S. Koivisto, "Linear growth of structure in massive bigravity," JCAP 1410, 066 (2014), arXiv:1404.4061 [astroph.CO]

[39] Macarena Lagos and Pedro G. Ferreira, "Cosmological perturbations in massive bigravity," JCAP 1412, 026 (2014), arXiv:1410.0207 [gr-qc]

[40] Frank Könnig and Luca Amendola, "Instability in a minimal bimetric gravity model," Phys.Rev. D90, 044030 (2014), arXiv:1402.1988 [astro-ph.CO].

[41] Frank Könnig, Yashar Akrami, Luca Amendola, Mariele Motta, and Adam R. Solomon, "Stable and unstable cosmological models in bimetric massive gravity," Phys.Rev. D90, 124014 (2014), arXiv:1407.4331 [astro-ph.CO].

[42] Jonas Enander, Yashar Akrami, Edvard Mörtsell, Malin Renneby, and Adam R. Solomon, "Integrated SachsWolfe effect in massive bigravity," Phys.Rev. D91, 
084046 (2015), arXiv:1501.02140 [astro-ph.CO]

[43] Frank Könnig, "Higuchi Ghosts and Gradient Instabilities in Bimetric Gravity," Phys.Rev. D91, 104019 (2015) arXiv:1503.07436 [astro-ph.CO]

[44] E. Mortsell and J. Enander, "Scalar instabilities in bimetric gravity: The Vainshtein mechanism and structure formation," JCAP 1510, 044 (2015), arXiv:1506.04977 [astro-ph.CO]
[45] Katsuki Aoki, Kei-ichi Maeda, and Ryo Namba, "Stability of the Early Universe in Bigravity Theory," Phys. Rev. D92, 044054 (2015), arXiv:1506.04543 [hep-th]

[46] Claudia de Rham, J. Tate Deskins, Andrew J. Tolley, and Shuang-Yong Zhou, "Graviton Mass Bounds," Rev. Mod. Phys. 89, 025004 (2017), arXiv:1606.08462 [astroph.CO] 